PROCEEDINGS OF THE

AMERICAN MATHEMATICAL SOCIETY

Volume 126, Number 7, July 1998, Pages 1955-1961

S 0002-9939(98)04337-8

\title{
SOME RESULTS ON FINITE DRINFELD MODULES
}

\author{
CHIH-NUNG HSU
}

(Communicated by William W. Adams)

\begin{abstract}
Let $\mathrm{K}$ be a global function field, $\infty$ a degree one prime divisor of $\mathrm{K}$ and let $\mathrm{A}$ be the Dedekind domain of functions in $\mathrm{K}$ regular outside $\infty$. Let $\mathrm{H}$ be the Hilbert class field of A, B the integral closure of $\mathrm{A}$ in $\mathrm{H}$. Let $\psi$ be a rank one normalized Drinfeld A -module and let $\mathfrak{P}$ be a prime ideal in $\mathrm{B}$. We explicitly determine the finite A-module structure of $\psi\left(\mathrm{B} / \mathfrak{P}^{N}\right)$. In particular, if $\mathrm{K}=\mathbb{F}_{q}(t), q$ is an odd prime number and $\psi$ is the Carlitz $\mathbb{F}_{q}[t]$-module, then the finite $\mathbb{F}_{q}[t]$-module $\psi\left(\mathbb{F}_{q}[t] / \mathfrak{P}^{N}\right)$ is always cyclic.
\end{abstract}

\section{INTRODUCTION}

Recall that $\mathrm{G}_{m}\left(\mathbb{Z} / p^{N} \mathbb{Z}\right)=\left(\mathbb{Z} / p^{N} \mathbb{Z}\right)^{\times}$is always cyclic except for the case that $p=2$ and $N \geq 3$; if $N \geq 3$, then $\left(\mathbb{Z} / 2^{N} \mathbb{Z}\right)^{\times}$is the direct product of two cyclic groups, one of order 2 , the other of order $2^{N-2}$. Let $X$ be a smooth, projective, geometrically connected curve defined over the finite field $\mathbb{F}_{q}$ with $q$ elements and let $\infty$ be a rational point on $X$. We set $\mathrm{K}$ to be the function field of $X$ over $\mathbb{F}_{q}$ and $\mathrm{A} \subset \mathrm{K}$ to be the Dedekind domain of functions regular outside $\infty$. We will consider the Drinfeld A-modules. From the view point of class field theory, these modules are interesting arithmetic objects over function fields. In particular, the rank one Drinfeld A-modules play a role entirely analogous to the important role played by $\mathrm{G}_{m}$ over number fields. This naturally leads us to explore an analogous phenomenon for rank one Drinfeld A-modules.

Let $\mathrm{K}_{\infty}$ be the completion of $\mathrm{K}$ with respective to $\infty$ and let $\mathrm{C}_{\infty}$ be the completion of the algebraic closure of $\mathrm{K}_{\infty}$ with respect to $\infty$. Let $\mathrm{C}_{\infty}\{\tau\}=\operatorname{End}_{\mathbb{F}_{q}}\left(\mathrm{G}_{a} / \mathrm{C}_{\infty}\right)$ be the twisted polynomial ring in the $q^{\text {th }}$ power Frobenius mapping $\tau$. A rank one Drinfeld A-module $\psi$ over $\mathrm{C}_{\infty}$ is an injective ring homomorphism $\psi: \mathrm{A} \rightarrow \mathrm{C}_{\infty}\{\tau\}$ such that the constant coefficient of $\psi_{a}(\tau)$ is equal to $a$ and $\operatorname{deg}_{\tau} \psi_{a}(\tau)=-\operatorname{Ord}_{\infty} a$ for all $a \in \mathrm{A}$. A sign-function (cf. [5] and [3]) $\operatorname{sgn}: \mathrm{K}_{\infty}^{\times} \rightarrow \mathbb{F}_{q}^{\times}$is a co-section of the inclusion map $\mathbb{F}_{q}^{\times} \hookrightarrow \mathrm{K}_{\infty}^{\times}$such that $\operatorname{sgn}(\alpha)=1$ for all $\alpha \in \mathrm{K}_{\infty}^{\times}$with $\alpha-1$ vanishing at $\infty$. A Drinfeld A-module $\psi$ of rank one over $\mathrm{C}_{\infty}$ is said to be sgn-normalized if the leading coefficient of $\psi_{a}(\tau)$ in $\tau$ is equal to $\operatorname{sgn}(a)$ for all $0 \neq a \in \mathrm{A}$. It is known [5] that any Drinfeld A-module of rank one over $\mathrm{C}_{\infty}$ is isomorphic to a sgn-normalized A-module $\psi$ over $\mathrm{H}$, where $\mathrm{H}$ is the Hilbert class field of $\mathrm{A}$, i.e., $\mathrm{H}$ is the maximal abelian extension of $\mathrm{K}$ such that the extension $\mathrm{H} / \mathrm{K}$ completely splits over $\infty$ and is unramified over every finite place of $\mathrm{K}$.

Received by the editors July 23, 1996 and, in revised form, December 26, 1996.

1991 Mathematics Subject Classification. Primary 11G09; Secondary 11A05.

Key words and phrases. Drinfeld modules, Hilbert class field.

(C)1998 American Mathematical Society 
Let $\mathrm{B}$ be the integral closure of $\mathrm{A}$ in $\mathrm{H}$ and let $\psi$ be a rank one sgn-normalized Drinfeld A-module. Let $\mathfrak{M}$ be an ideal in B. Via the action of $\psi(\bmod \mathfrak{M}), \mathrm{B} / \mathfrak{M}$ becomes a finite A-module. We denote this by $\psi(\mathrm{B} / \mathfrak{M})$. This finite A-module plays the role of $\mathrm{G}_{m}\left(\mathbb{Z} / p^{N} \mathbb{Z}\right)$. The purpose of this note is to determine the Amodule structure of $\psi(\mathrm{B} / \mathfrak{M})$. It is sufficient to consider the case $\psi\left(\mathrm{B} / \mathfrak{P}^{N}\right)$, where $\mathfrak{P}$ is a prime ideal in $\mathrm{B}$. The structure of $\psi\left(\mathrm{B} / \mathfrak{P}^{N}\right)$ is obtained in Theorems 2.1 and 2.2. In particular, if $\psi$ is the Carlitz $\mathbb{F}_{q}[t]$-module ([3], chapter 3$)$ and $q \neq 2$, then $\psi\left(\mathrm{B} / \mathfrak{P}^{N}\right)$ is always cyclic (cf. Corollaries 2.1 and 2.2). The Carlitz case is completely analogous to the classical case.

In section 3 , we discuss the relations between $\mathrm{B}_{\mathfrak{P}}$ and $\varliminf_{\lfloor} \psi\left(\mathrm{B} / \mathfrak{P}^{N}\right)$ via the exponential and logarithm functions of the sgn-normalized Drinfeld module $\psi$.

\section{The Structure of $\psi\left(\mathrm{B} / \mathfrak{P}^{N}\right)$}

Let the notation $X, \mathbb{F}_{q}, \infty, \mathrm{K}, \mathrm{A}, \mathrm{H}, \mathrm{B}$ and sgn be as in the introduction. If $\mathrm{M}$ is a commutative $\mathbb{F}_{q}$-algebra, we let $\mathrm{M}\{\tau\}$ denote the composition ring of Frobenius polynomials in $\tau$, where $\tau$ is the $q^{\text {th }}$ power mapping. From now on, we let $\psi$ be a sgn-normalized rank one Drinfeld A-module over $\mathrm{H}$, i.e., $\psi: \mathrm{A} \rightarrow \mathrm{H}\{\tau\}$ is a rank one Drinfeld A-module over $\mathrm{H}$ such that for any $a \in \mathrm{A}$, the leading coefficient of $\psi_{a}(\tau)$ is equal to $\operatorname{sgn}(a)$. It is known that $\psi_{a}(\tau) \in \mathrm{B}\{\tau\}$ for all $a \in \mathrm{A}$. Thus, via $\psi, \mathrm{B}$ becomes an $\mathrm{A}$-module. We denote this module by $\psi(\mathrm{B})$ and denote the action $\psi_{a}(\tau)(b)$ by $b^{a}$ for all $a \in \mathrm{A}, b \in \mathrm{B}$. Let $\mathfrak{M}$ be an ideal in B. Since $\psi(\mathrm{A}) \subset \mathrm{B}\{\tau\}$, it follows that, via $\psi(\bmod \mathfrak{M}), \mathrm{B} / \mathfrak{M}$ becomes a finite A-module. We denote this finite module by $\psi(\mathrm{B} / \mathfrak{M})$. If the decomposition of $\mathfrak{M}$ is equal to $\mathfrak{P}_{1}^{N_{1}} \mathfrak{P}_{2}^{N_{2}} \cdots \mathfrak{P}_{L}^{N_{L}}$, where $\mathfrak{P}_{1}, \mathfrak{P}_{2}, \cdots, \mathfrak{P}_{L}$ are prime ideals in $\mathrm{B}$, then, by the Chinese remainder theorem, we have

$$
\psi(\mathrm{B} / \mathfrak{M}) \cong \bigoplus_{i=1}^{L} \psi\left(\mathrm{B} / \mathfrak{P}_{i}^{N_{i}}\right) .
$$

Thus to determine the A-module structure of $\psi(\mathrm{B} / \mathfrak{M})$, it is sufficient to consider the case $\psi\left(\mathrm{B} / \mathfrak{P}^{N}\right)$, where $\mathfrak{P}$ is a prime ideal in $\mathrm{B}$. Let $\wp=\mathfrak{P} \cap \mathrm{A}$ and let $f$ be the dimension of the vector space $\mathrm{B} / \mathfrak{P}$ over $\mathrm{A} / \wp$. It follows from class field theory that $\operatorname{Norm}_{\mathrm{K}}^{\mathrm{H}} \mathfrak{P}=\wp^{f}$ is a principal ideal in $\mathrm{A}$. We let $\wp^{f}=\left(\pi_{\wp}\right)$ for the unique element $\pi_{\wp} \in \mathrm{A}$ with $\operatorname{sgn}\left(\pi_{\wp}\right)=1$. It is known that $\psi(\mathrm{B} / \mathfrak{P})$ is a cyclic A-module with Euler-Poincaré characteristic $\pi_{\wp}-1$ (cf. [3], chapter 4), i.e., as A-module,

$$
\psi(\mathrm{B} / \mathfrak{P}) \cong \mathrm{A} /\left(\pi_{\wp}-1\right) \text {. }
$$

We let $\psi_{\wp}(\tau)$ be the monic generator of the left ideal of $\mathrm{H}\{\tau\}$ generated by $\psi_{a}(\tau)$ for all $a \in \wp$. We also denote the polynomial $\psi_{\wp}(\tau)(x)$ in $x$ by $x^{\wp}$ for all $x \in \mathrm{H}$. The important property of the polynomial $\psi_{\wp}(\tau), \wp \subset \mathrm{A}$ a prime ideal, is the following (cf. [5], Proposition 11.4):

$$
f(x)=\psi_{\wp}(\tau)(x) / x=x^{\wp} / x
$$

is an Eisenstein polynomial over B at any prime ideal $\mathfrak{P}$ above $\wp$. Let $c_{\wp}=f(0) \in \mathrm{B}$. Then we have $\operatorname{deg}_{\mathfrak{P}} c_{\wp}=1$. If $\wp$ is a rational point on the curve $X$ (i.e., $\wp$ is a prime ideal in $\mathrm{A}$ of degree one) defined over $\mathbb{F}_{2}$, then $\psi_{\wp}(\tau)=c_{\wp} \tau^{0}+\tau^{1} \in \mathrm{B}\{\tau\}$, i.e., $x^{\wp}=\psi_{\wp}(\tau)(x)=c_{\wp} x+x^{2}$, where $c_{\wp} \in \mathfrak{P}$ but $c_{\wp} \notin \mathfrak{P}^{2}$.

Lemma 2.1. Suppose that $N$ is a positive integer, $\mathfrak{P}$ and $\wp$ are as above. Then for any $b_{1}, b_{2} \in \psi(\mathrm{B})$, if $b_{1} \equiv b_{2}\left(\bmod \mathfrak{P}^{N}\right)$, then $b_{1}^{\wp} \equiv b_{2}^{\wp}\left(\bmod \mathfrak{P}^{N+1}\right)$. 
Proof. We may write $b_{1}=b_{2}+x$ for some $x \in \mathfrak{P}^{N}$. Then, by the Eisenstein polynomial property,

$$
b_{1}^{\wp}=\left(b_{2}+x\right)^{\wp}=b_{2}^{\wp}+x^{\wp} \equiv b_{2}^{\wp} \quad\left(\bmod \mathfrak{P}^{N+1}\right) .
$$

Lemma 2.2. Suppose that $\mathfrak{P}, \wp$ and $c_{\wp}$ are as above. Then:

(1) If $N \geq 2$ is a positive integer, then $x^{\wp^{N-2}} \equiv x \cdot c_{\wp}^{N-2}\left(\bmod \mathfrak{P}^{N}\right)$ for all $x \in \mathfrak{P}$ except for the case that $\wp$ is a rational point on the curve $X$ defined over $\mathbb{F}_{2}$.

(2) If $\wp$ is a rational point on the curve $X$ defined over $\mathbb{F}_{2}$ (i.e., $\wp$ is a prime ideal of A of degree one) and $N \geq 3$ is a positive integer, then

$$
x^{\wp^{N-3}} \equiv x \cdot c_{\wp}^{N-3} \quad\left(\bmod \mathfrak{P}^{N}\right)
$$

for all $x \in \mathfrak{P}^{2}$.

(3) If $\wp$ is a rational point on the curve $X$ defined over $\mathbb{F}_{2}$, then for all $x \in \mathfrak{P}, x \not \equiv$ $0, c_{\wp}\left(\bmod \mathfrak{P}^{2}\right)$, we have $x^{\wp} \in \mathfrak{P}^{2}$ but $x^{\wp} \notin \mathfrak{P}^{3}$.

Proof. Assertion (1) is obvious for $N=2$. Now suppose that it is true for $N \geq 2$, i.e.,

$$
x^{\wp^{N-2}} \equiv x \cdot c_{\wp}^{N-2} \quad\left(\bmod \mathfrak{P}^{N}\right) .
$$

We show that it is true for $N+1$. Applying Lemma 2.1 and the Eisenstein polynomial property, we have

$$
\left(x^{\wp^{N-2}}\right)^{\wp} \equiv\left(x \cdot c_{\wp}^{N-2}\right)^{\wp} \equiv c_{\wp} \cdot\left(x \cdot c_{\wp}^{N-2}\right)+\left(x \cdot c_{\wp}^{N-2}\right)^{q^{\operatorname{deg} \wp}} \quad\left(\bmod \mathfrak{P}^{N+1}\right) .
$$

If $q \neq 2$ or $q=2$ and $\wp$ is not a rational point on the curve $X$ over $\mathbb{F}_{2}$ (i.e., $\left.q^{\operatorname{deg} \wp} \geq 3\right)$, then we have $\left(x \cdot c_{\wp}^{N-2}\right)^{q^{\operatorname{deg} \wp}} \in \mathfrak{P}^{N+1}$. Hence

$$
x^{\wp^{N-1}} \equiv x c_{\wp}^{N-1} \quad\left(\bmod \mathfrak{P}^{N+1}\right) .
$$

This completes the proof of (1). The proof of (2) is similar.

To prove (3), since $x \in \mathfrak{P}$ and $\operatorname{deg}_{\mathfrak{P}} c_{\wp}=1, x^{\wp}=c_{\wp} \cdot x+x^{2}=x\left(x+c_{\wp}\right) \in \mathfrak{P}^{2}$. The assertion $x^{\wp} \notin \mathfrak{P}^{3}$ follows from the facts that the characteristic of $\mathrm{B}$ is 2 and $x \not \equiv 0, c_{\wp}\left(\bmod \mathfrak{P}^{2}\right)$.

Lemma 2.3. Suppose that $\mathfrak{P}$ and $\wp$ are as above. Then:

(1) If $N \geq 2$ is a positive integer and $x \in \mathfrak{P}, x \notin \mathfrak{P}^{2}$, then the submodule $\langle\bar{x}\rangle$ of $\psi\left(\mathrm{B} / \mathfrak{P}^{N}\right)$ generated by $\bar{x}=x\left(\bmod \mathfrak{P}^{N}\right) \in \psi\left(\mathrm{B} / \mathfrak{P}^{N}\right)$ is isomorphic to $\mathrm{A} / \wp^{N-1}$ except for the case when $\wp$ is a rational point on the curve $X$ defined over $\mathbb{F}_{2}$.

(2) Suppose that $\wp$ is a rational point on the curve $X$ defined over $\mathbb{F}_{2}$. If $N \geq 3$ is a positive integer and $x \in \mathfrak{P}^{2}, x \notin \mathfrak{P}^{3}$, then the submodule $\langle\bar{x}\rangle$ of $\psi\left(\mathrm{B} / \mathfrak{P}^{N}\right)$ generated by $\bar{x}=x\left(\bmod \mathfrak{P}^{N}\right) \in \psi\left(\mathrm{B} / \mathfrak{P}^{N}\right)$ is isomorphic to $\mathrm{A} / \wp^{N-2}$.

(3) Suppose that $\wp$ is a rational point on the curve $X$ defined over $\mathbb{F}_{2}$ and $N \geq 2$. Then for all $x \in \mathfrak{P}, x \not \equiv 0, c_{\wp}\left(\bmod \mathfrak{P}^{2}\right)$, the submodule $\langle\bar{x}\rangle$ of $\psi\left(\mathrm{B} / \mathfrak{P}^{N}\right)$ generated by $\bar{x}=x\left(\bmod \mathfrak{P}^{N}\right) \in \psi\left(\mathrm{B} / \mathfrak{P}^{N}\right)$ is isomorphic to $\mathrm{A} / \wp^{N-1}$.

Proof. By Lemma $2.2(1), x^{\wp^{N-1}} \equiv x \cdot c_{\wp}^{N-1}\left(\bmod \mathfrak{P}^{N+1}\right)$. Since $\operatorname{deg}_{\mathfrak{P}} c_{\wp}=1$ and $x \in \mathfrak{P}$, we have

$$
x^{\wp^{N-1}} \equiv 0 \quad\left(\bmod \mathfrak{P}^{N}\right)
$$


Again by Lemma $2.2(1), \operatorname{deg}_{\mathfrak{P}} c_{\wp}=1$ and $x \in \mathfrak{P}, x \notin \mathfrak{P}^{2}$,

$$
x^{\wp^{N-2}} \equiv x \cdot c_{\wp}^{N-2} \not \equiv 0 \quad\left(\bmod \mathfrak{P}^{N}\right) .
$$

Since $\langle\bar{x}\rangle$ is a cyclic A-module, $\langle\bar{x}\rangle$ is isomorphic to $\mathrm{A} / \wp^{N-1}$. The proof of (2) is similar, and the proof of (3) follows from Lemma $2.2(3),(2)$.

Let $[\mathrm{H}: \mathrm{K}]=f r$ be the class number of $\mathrm{A}$, where $f=[\mathrm{B} / \mathfrak{P}: \mathrm{A} / \wp]$. Then the main result is

Theorem 2.1. Suppose that $\mathfrak{P}, \wp, \pi_{\wp}$ and $f$ are as above. Then

$$
\psi\left(\mathrm{B} / \mathfrak{P}^{N}\right) \cong \begin{cases}\mathrm{A} /\left(\pi_{\wp}-1\right) & \text { if } N=1, \\ \mathrm{~A} /\left(\pi_{\wp}-1\right) \oplus\left(\mathrm{A} / \wp^{N-1}\right)^{f} & \text { if } N>1,\end{cases}
$$

except for the case when $\wp$ is a rational point on the curve $X$ defined over $\mathbb{F}_{2}$.

Proof. The case $N=1$ follows from the theory of Drinfeld modules over finite fields (cf. [3], Chapter 4). We suppose that $N \geq 2$. Given $x \in \psi(B)$. Since $\psi(\mathrm{B} / \mathfrak{P}) \cong \mathrm{A} /\left(\pi_{\wp}-1\right)$, it follows that $x^{\pi_{\wp}-1} \equiv 0(\bmod \mathfrak{P})$. By Lemma $2.2(1)$, we have

$$
\left(x^{\pi_{\wp}-1}\right)^{\wp^{N-1}} \equiv 0 \quad\left(\bmod \mathfrak{P}^{N}\right) .
$$

This implies that the Euler-Poincaré characteristic of any A-cyclic submodule of $\psi\left(\mathrm{B} / \mathfrak{P}^{N}\right)$ divides $\left(\pi_{\wp}-1\right) \wp^{N-1}$.

Since $\psi(\mathrm{A}) \subset \mathrm{B}\{\tau\}, \psi\left(\mathfrak{P} / \mathfrak{P}^{N}\right)$ is a submodule of $\psi\left(\mathrm{B} / \mathfrak{P}^{N}\right)$. We have

$$
\operatorname{dim}_{\mathbb{F}_{q}} \psi\left(\mathfrak{P} / \mathfrak{P}^{N}\right)=(N-1) \operatorname{dim}_{\mathbb{F}_{q}} \mathrm{~B} / \mathfrak{P}=f(N-1) \operatorname{dim}_{\mathbb{F}_{q}} \mathrm{~A} / \wp .
$$

Since $x^{\wp^{N-1}} \equiv 0\left(\bmod \mathfrak{P}^{N}\right)$ for all $x \in \mathfrak{P}($ by Lemma2.2 $(1))$, as A-module

$$
\psi\left(\mathfrak{P} / \mathfrak{P}^{N}\right) \cong \bigoplus_{i=1}^{l} \mathrm{~A} / \wp^{n_{i}}
$$

for suitable positive integers $1 \leq n_{1} \leq n_{2} \leq \cdots \leq n_{l} \leq N-1$ such that

$$
n_{1}+n_{2}+\cdots+n_{l}=(N-1) f .
$$

By Lemma $2.2(1)$ and Lemma $2.3(1)$, the subset of elements $\bar{x}$ in $\psi\left(\mathfrak{P} / \mathfrak{P}^{N}\right)$ such that $x^{\wp^{N-2}} \equiv 0\left(\bmod \mathfrak{P}^{N}\right)$ is equal to $\psi\left(\mathfrak{P}^{2} / \mathfrak{P}^{N}\right)$. Counting cardinalities, we must have

$$
l=f, \quad n_{1}=n_{2}=\cdots=n_{f}=N-1 .
$$

Thus we get

$$
\psi\left(\mathfrak{P} / \mathfrak{P}^{N}\right) \cong\left(\mathrm{A} / \wp^{N-1}\right)^{f} .
$$

Next, we take $g \in \mathrm{B}$ such that $g(\bmod \mathfrak{P})$ is a generator of $\psi(\mathrm{B} / \mathfrak{P})$. We let $\langle\bar{g}\rangle$ be the A-submodule of $\psi\left(\mathrm{B} / \mathfrak{P}^{N}\right)$ generated by $\bar{g}=g\left(\bmod \mathfrak{P}^{N}\right)$ in $\psi\left(\mathrm{B} / \mathfrak{P}^{N}\right)$. We define the A-module homomorphism $\chi:\langle\bar{g}\rangle \rightarrow \psi(\mathrm{B} / \mathfrak{P})$ by $\chi\left(x\left(\bmod \mathfrak{P}^{N}\right)\right)=x$ $(\bmod \mathfrak{P})$ for all $x\left(\bmod \mathfrak{P}^{N}\right)$ in $\langle\bar{g}\rangle$. Since $g(\bmod \mathfrak{P})$ is a generator of $\psi(\mathrm{B} / \mathfrak{P})$ and $\chi\left(g\left(\bmod \mathfrak{P}^{N}\right)\right)=g(\bmod \mathfrak{P}), \chi$ is a surjective homomorphism of $\langle\bar{g}\rangle$ onto $\psi(\mathrm{B} / \mathfrak{P})$. This implies that $\left(\pi_{\wp}-1\right)$ divides the Euler-Poincaré characteristic of $\psi\left(\mathrm{B} / \mathfrak{P}^{N}\right)$, because $\langle\bar{g}\rangle$ is a submodule of $\psi\left(\mathrm{B} / \mathfrak{P}^{N}\right)$. Combining these, we obtain that $\left(\pi_{\wp}-1\right) \wp^{f(N-1)}$ divides the Euler-Poincaré characteristic of $\psi\left(\mathrm{B} / \mathfrak{P}^{N}\right)$; this implies that the Euler-Poincaré characteristic of $\psi\left(\mathrm{B} / \mathfrak{P}^{N}\right)$ is equal to 
$\left(\pi_{\wp}-1\right) \wp^{f(N-1)}$; hence $\langle\bar{g}\rangle$ (or $\left.\psi\left(\mathrm{B} / \mathfrak{P}^{N}\right)\right)$ contains an A-submodule which is isomorphic to $\mathrm{A} /\left(\pi_{\wp}-1\right)$. Therefore, we obtain that

$$
\psi\left(\mathrm{B} / \mathfrak{P}^{N}\right) \cong \mathrm{A} /\left(\pi_{\wp}-1\right) \oplus\left(\mathrm{A} / \wp^{N-1}\right)^{f} .
$$

Theorem 2.2. Suppose that $\wp$ is a rational point on the curve $X$ defined over $\mathbb{F}_{2}$. Then

$$
\psi\left(\mathrm{B} / \mathfrak{P}^{N}\right) \cong \begin{cases}\mathrm{A} /\left(\pi_{\wp}-1\right), & \text { if } N=1 ; \\ \mathrm{A} /\left(\pi_{\wp}-1\right) \oplus(\mathrm{A} / \wp)^{f}, & \text { if } N=2 ; \\ \mathrm{A} /\left(\pi_{\wp}-1\right) \oplus \mathrm{A} / \wp \oplus\left(\mathrm{A} / \wp^{N-1}\right)^{f-1} \oplus \mathrm{A} / \wp^{N-2}, & \text { if } N \geq 3 .\end{cases}
$$

Proof. The case $N=1$ is standard. We suppose that $N \geq 2$. Using Lemmas 2.2 and 2.3, the proof is almost the same as the proof of Theorem 2.1. We obtain that the Euler-Poincaré characteristic of any A-cyclic submodule of $\psi\left(\mathrm{B} / \mathfrak{P}^{N}\right)$ divides $\left(\pi_{\wp}-1\right) \wp^{N-1}$, the finite A-module $\psi\left(\mathfrak{P} / \mathfrak{P}^{N}\right)$ is annihilated by $\wp^{N-1}$, and $\psi\left(\mathrm{B} / \mathfrak{P}^{N}\right)$ contains an $\mathrm{A}$-submodule which is isomorphic to $\mathrm{A} /\left(\pi_{\wp}-1\right)$. From these, we deduce that the Euler-Poincaré characteristic of $\psi\left(\mathrm{B} / \mathfrak{P}^{N}\right)$ is equal to $\left(\pi_{\wp}-1\right) \wp^{f(N-1)}$ and

$$
\psi\left(\mathrm{B} / \mathfrak{P}^{N}\right) \cong \mathrm{A} /\left(\pi_{\wp}-1\right) \oplus \psi\left(\mathfrak{P} / \mathfrak{P}^{N}\right) .
$$

Next, we deal with the A-module structure of $\psi\left(\mathfrak{P} / \mathfrak{P}^{N}\right)$. For $N=2$, since $\psi\left(\mathfrak{P} / \mathfrak{P}^{2}\right)$ is annihilated by $\wp$, counting the dimension of $\psi\left(\mathfrak{P} / \mathfrak{P}^{2}\right)$ over $\mathrm{A} / \wp$, we obtain that $\psi\left(\mathfrak{P} / \mathfrak{P}^{2}\right) \cong(\mathrm{A} / \wp)^{f}$. For $N \geq 3$, since $\wp$ is a rational point on the curve $X$ defined over $\mathbb{F}_{2}$ and $[\mathrm{B} / \mathfrak{P}: \mathrm{A} / \wp]=f$, as abelian group $\mathfrak{P} / \mathfrak{P}^{2} \cong(\mathrm{A} / \wp)^{f} \cong \mathbb{F}_{2}^{f}$. Let $S$ be the A-submodule of $\psi\left(\mathfrak{P} / \mathfrak{P}^{N}\right)$ generated by elements $x\left(\bmod \mathfrak{P}^{N}\right)$ is such that $x \in \mathfrak{P}$ but $x \notin \mathfrak{P}^{2}$. We define the abelian group homomorphism $\chi: S \rightarrow$ $\psi\left(\mathfrak{P}^{2} / \mathfrak{P}^{3}\right)$ by $\chi\left(x\left(\bmod \mathfrak{P}^{N}\right)\right)=x^{\wp}\left(\bmod \mathfrak{P}^{3}\right)$ for all $x\left(\bmod \mathfrak{P}^{N}\right) \in S$. From Lemma $2.2(3)$, we know that if $x\left(\bmod \mathfrak{P}^{N}\right) \in S$ is such that $\chi\left(x\left(\bmod \mathfrak{P}^{N}\right)\right)=0$, then $x \equiv 0, c_{\wp}\left(\bmod \mathfrak{P}^{2}\right)$. This implies that $\operatorname{dim}_{\mathbb{F}_{2}} \chi(S)=f-1$. Combining this with Lemma $2.3(3)$ and the fact that $S$ is annihilated by $\wp^{N-1}$, we obtain that

$$
S \cong \mathrm{A} / \wp \oplus\left(\mathrm{A} / \wp^{N-1}\right)^{f-1} .
$$

Since $\chi\left(c_{\wp}\left(\bmod \mathfrak{P}^{N}\right)\right)=0$ and $\left.c_{\wp}\left(\bmod \mathfrak{P}^{N}\right)\right) \in S$, there exists an element $x \in \mathfrak{P}^{2}, x \notin \mathfrak{P}^{3}$, such that $x\left(\bmod \mathfrak{P}^{3}\right) \notin \chi(S)$. By Lemma $2.2(2)$, we know that $\left\langle x\left(\bmod \mathfrak{P}^{N}\right)\right\rangle$ is a submodule of $\psi\left(\mathfrak{P} / \mathfrak{P}^{N}\right)$ which is isomorphic to $\mathrm{A} / \wp^{N-2}$. Combining these and counting the dimension of $\psi\left(\mathfrak{P} / \mathfrak{P}^{N}\right)$ over $\mathrm{A} / \wp \cong \mathbb{F}_{2}$, we obtain that

$$
\psi\left(\mathrm{B} / \mathfrak{P}^{N}\right) \cong \mathrm{A} /\left(\pi_{\wp}-1\right) \oplus \mathrm{A} / \wp \oplus\left(\mathrm{A} / \wp^{N-1}\right)^{f-1} \oplus \mathrm{A} / \wp^{N-2} .
$$

This completes the proof.

As an application, we let $\mathrm{A}=\mathbb{F}_{q}[t]$ and let $\phi$ be the Carlitz A-module, i.e., $\phi: \mathrm{A} \rightarrow \mathbb{F}_{2}(t)\{\tau\}$ is given by

$$
\phi_{t}(\tau)=t \tau^{0}+\tau^{1} .
$$

Then we have

Corollary 2.1. If $N$ is a positive integer and $\wp=(p)$ is a prime ideal in A generated by the monic polynomial $p$, then the finite A-module

$$
\phi\left(\mathrm{A} / \wp^{N}\right) \cong \mathrm{A} /\left(p^{N}-p^{N-1}\right)
$$

is cyclic except for the case when $\mathbb{F}_{q}$ equals $\mathbb{F}_{2}$ and $p \mid t(t+1)$. 
Corollary 2.2. If $N$ is a positive integer, $\mathrm{A}=\mathbb{F}_{2}[t], \wp=(p)$ with $p=t$ or $t+1 \in \mathrm{A}$, then the finite $\mathrm{A}$-module $\phi\left(\mathrm{A} / \wp^{N}\right)$ is isomorphic to

$$
\begin{cases}\mathrm{A} /(p-1), & \text { if } N=1 \\ \mathrm{~A} /\left(t^{2}+t\right), & \text { if } N=2 \\ \mathrm{~A} /\left(t^{2}+t\right) \oplus \mathrm{A} /\left(p^{N-2}\right) & \text { if } N \geq 3\end{cases}
$$

\section{Passage to the limit}

Let the notation $X, \mathbb{F}_{q}, \infty, \mathrm{K}, \mathrm{A}, \mathrm{H}, \mathrm{B}, f, \pi_{\wp}$ and sgn be as before. Let $\psi$ be a sgn-normalized rank one Drinfeld A-module over H. Suppose that $\mathfrak{P}$ and $\wp$ are as in section 2 and $\wp$ does not correspond to a rational point on $X$ if $q=2$. It is well-know that there exists a lattice $\mathfrak{A} \zeta, \zeta \in \mathrm{C}_{\infty}, \mathfrak{A}$ an ideal of $\mathrm{A}$, of rank one such that $\psi$ is determined by this lattice. The exponential function $e_{\psi}$ associated to $\mathfrak{A} \zeta$ is defined to be

$$
e_{\psi}(x)=z \prod_{a \in \mathfrak{A}}\left(1-\frac{x}{a \cdot \zeta}\right) \in \mathrm{H}\{\{\tau\}\} .
$$

Let $\mathrm{H}_{\mathfrak{P}}\left(\right.$ resp. $\mathrm{K}_{\wp}$ ) be the completion fields associated to $\mathfrak{P}($ resp. $\wp)$. Let $\mathrm{B}_{\mathfrak{P}} \subset \mathrm{H}_{\mathfrak{P}}$ and $\mathrm{A}_{\wp} \subset \mathrm{K}_{\wp}$ be the rings of integers.

It follows from theorem 2.1 that as A-module

$$
\begin{aligned}
\psi\left(\mathrm{B}_{\mathfrak{P}}\right) & =\psi\left(\varliminf_{\lim } \mathrm{B} / \mathfrak{P}^{N}\right) \\
& =\longleftarrow \psi\left(\mathrm{B} / \mathfrak{P}^{N}\right) \\
& =\varliminf_{\mathrm{lim}} \mathrm{A} /\left(\pi_{\wp}-1\right) \oplus\left(\mathrm{A} / \wp^{N-1}\right)^{f} \\
& =\mathrm{A} /\left(\pi_{\wp}-1\right) \oplus \mathrm{A}_{\wp}^{f} \\
& =\mathrm{A} /\left(\pi_{\wp}-1\right) \oplus \mathrm{B}_{\mathfrak{P}} .
\end{aligned}
$$

We know that the coefficients of $e_{\psi}$ are in $\mathrm{H}$ and these coefficients are obtained by solving a recursion equation via any $\psi_{a}, a \in \mathrm{A}, a \notin \mathbb{F}_{q}$ (cf. [3], Lemma 4.6.5). We can deduce from this recursion that $e_{\psi}$ converges in a neighborhood of 0 . Thus there exist element $\alpha \in \mathrm{H}_{\mathfrak{P}}$ such that $e(x)=e_{\psi}(\alpha \cdot x)$ is an analytic injective function of $\mathrm{B}_{\mathfrak{P}}$ into $\mathrm{B}_{\mathfrak{P}}$. By the property of the exponential function $e_{\psi}$, we obtain that $e(a x)=\psi_{a}(e(x))$ for all $a \in \mathrm{A}$. Combining these, we have

Theorem 3.1. As A-module,

$$
\psi\left(\mathrm{B}_{\mathfrak{P}}\right) \cong \begin{cases}\mathrm{A} /\left(\pi_{\wp}-1\right) \oplus \mathrm{A} / \wp \oplus \mathrm{B}_{\mathfrak{P}} & \text { if } q=2 \text { and } \wp \text { is a rational point } \\ \mathrm{A} /\left(\pi_{\wp}-1\right) \oplus \mathrm{B}_{\mathfrak{P}} & \text { otherwise. }\end{cases}
$$

Moreover, one has an analytic map $e: \mathrm{B}_{\mathfrak{P}} \rightarrow \psi\left(\mathrm{B}_{\mathfrak{P}}\right)$ satisfying the following commutative diagram:

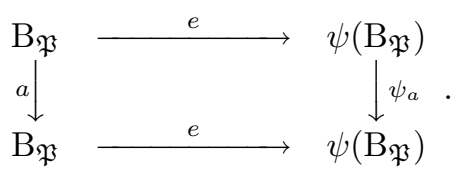




\section{REFERENCES}

[1] E. -U. Gekeler 'Drinfeld Modular Curves', LNM 1231, Springer-Verlag (1986). MR 88b:11077

[2] E. -U. Gekeler 'On finite Drinfeld Modules', J. Algebra 141 (1991), pp. 187-203. MR 92e: 11064

[3] David Goss 'Basic Structures of Function Field Arithmetic', Springer-Verlag (1996). CMP 97:05

[4] D. R. Hayes 'Explicit class field theory for ration function fields', Transations of the American Mathematical Society, vol 189 (1974), pp. 77 - 91. MR 48:8444

[5] D. R. Hayes 'A Brief introduction to Drinfeld modules', in 'The Arithmetic of Function Fields' (edited by D. Goss, D. R. Hayes and M. I. Rosen), (1992), pp. 1-32. MR 93m:11050

[6] K. Ireland and M. Rosen 'A Classical Introduction to Modern Number Theory', SpringerVerlag. MR 92e:11001

[7] J. K. Yu 'Isogenies of Drinfeld modules over finite fields', J. Number Theory 54 (1995), pp. 161-171. MR 96i: 11060

Department of Mathematics, National Taiwan Normal University, 88 Sec. 4 TingCHOU RoAD, TAIPEI, TAIWAN

E-mail address: maco@math.ntnu.edu.tw 